\title{
Riscos dos agroquímicos para a saúde humana
}

\author{
Maria Hosana Conceição ${ }^{l}$
}

\begin{abstract}
RESUMO - A presença de resíduos de agroquímicos em alimentos é um assunto que aflige consumidores e órgãos de saúde devido ao potencial risco à saúde que pode advir da exposição humana a esses compostos. Nesse trabalho, relatamos a avaliação da exposição humana crônica aos fungicidas ditiocarbamatos em 180 amostras de tomates do comércio do Distrito Federal.
\end{abstract}

Palavras-chave: agroquímicos, alimentos, saúde.

\section{Agrochemicals risks to human health}

\begin{abstract}
The presence of agrochemicals residues in food is a serious problems to consumers and health agency just to the potential human risk that can result from the exposure to the compounds. In this work, the evaluation of chronic human exposure to dithiocarbamates fungicides was studied in the one hundred and eighty tomato samples collected at the Federal District marked.
\end{abstract}

Key words: agrochemicals, food, health.

A toxicidade e o modo de ação dos defensivos agrícolas no homem e nos animais variam largamente, estando diretamente relacionados com sua estrutura química. Um exemplo são os inseticidas organofosforados e carbamatos, estruturalmente relacionados com o neurotransmissor acetilcolina, e que inibem a ação da enzima do tecido nervoso acetil colinesterase, responsável pela destruição e terminação da atividade biológica do neurotransmissor. Aproximadamente $20 \%$ de todos os pesticidas conhecidos são suspeitos de serem carcinogênicos. Além desses efeitos adversos, os pesticidas podem ser teratogênicos e mutagênicos, podendo afetar o sistema imunológico (Ecobichon, 1996).

O uso de agroquímicos no campo atinge primordialmente os trabalhadores rurais, que manuseiam e aplicam estes compostos. A Organização Mundial de

1 Professora Dra. daFaculdade de Ciências da Saúde-FACS do UniCEUB. 
Saúde estima que ocorreram no mundo até 1990 cerca de três milhões de intoxicações agudas causadas por esses compostos, com cerca de 220 mil mortes por ano. Cerca de $70 \%$ dos casos registrados ocorreram em países em desenvolvimento (Jeyaratnam, 1990).

A Agência de Proteção Ambiental estima que só nos Estados Unidos ocorrem de 10.000 a 20.000 diagnósticos clínicos de intoxicação por agroquímicos por ano, entre 3.380.000 trabalhadores rurais, fumigadores e outras ocupações (WHO, 2002). O uso de equipamentos de proteção adequados pelo agricultor pode reduzir em até $100 \%$ a exposição (Bonsal, 1985). Entretanto, devido a questões econômicas, culturais ou desinformação quanto ao risco, o uso desses equipamentos, muitas vezes, é precário ou inexistente.

O Grupo de Estudos Epidemiológicos em Toxicologia, formado pelos centros de Belo Horizonte, Campinas, Florianópolis, Londrina, Maringá e Ribeirão Preto, relatou que os pesticidas foram responsáveis por 46,9\% das mortes nos seis Hospitais Universitários do Brasil no período de 1994-1996, dentre os 53921 casos de intoxicação, no campo, relatados (Zambrone, 1999). De acordo com os dados do Sistema Integrado de Informação Toxicológica (SINITOX), 18 óbitos causados por agrotóxicos ocorreram em 1999 na região Centro-Oeste (14 do sexo masculino), correspondendo a 38\% de todas as mortes causadas por agentes tóxicos na região (Sinitox, 2002).

Enquanto a população rural está sob risco de exposição aguda, o consumidor de alimentos se encontra sob risco da exposição crônica aos agroquímicos, pela ingestão de alimentos contendo resíduos desses compostos. Nos Estados Unidos, pesquisas de opinião revelaram que cerca de $80 \%$ dos consumidores consideram a presença de resíduos de agroquímicos em alimentos um problema de saúde pública (Winter, 1992). Doll \& Petto (1981) estimaram que aproximadamente $35 \%$ de toda mortalidade por câncer nos Estados Unidos está relacionado com a dieta. Órgãos governamentais americanos estimaram que a cada ano, aproximadamente 6.000 crianças, em idade pré-escolar, podem adquirir câncer em consequência da exposição a resíduos de pesticidas encontrados em frutas e vegetais (Winter, 1992).

Indicadores de toxicidade dos agroquímicos estabelecidos internacionalmente incluem a dose letal, a classe toxicológica e a ingestão diária aceitável (IDA). A dose letal $\left(\mathrm{DL}_{50}\right)$ representa a dose de um pesticida que causa a morte de $50 \%$ de uma população de animais de laboratório. $\mathrm{A} \mathrm{DL}_{50}$ pode variar significativamente entre as espécies testadas e depende, em grande parte, das condições experimentais do estudo (Ecobichon, 1996). A classe toxicológica de um agroquímico está diretamente relacionada com a dose letal média do composto numa dada formulação podendo um pesticida estar enquadrado em diferentes 
classes toxicológicas dependendo da formulação em que se encontra. Enquanto a dose letal é um índice de toxicidade aguda em animais de laboratório, a classe toxicológica é um parâmetro de toxicidade aguda no homem. Os produtos agrotóxicos podem ser classificados como pertencentes à classe I, a mais tóxica e às classes II, III e IV, menos tóxicas. A obrigatoriedade de indicação na rotulagem dos produtos da classe toxicológica a que pertencem é essencial para orientar os agricultores sobre os cuidados necessários durante seu uso.

A IDA, ou também chamada de dose de referência (RfD), representa a dose ingerida pelo homem por toda a vida sem que ocorra efeito adverso apreciável à saúde, representando um parâmetro de toxicidade crônica para o homem. Este valor, em $\mathrm{mg} / \mathrm{kg}$ peso corpóreo / dia, é estabelecido para cada pesticida baseado em estudos toxicológicos crônicos conduzidos em animais de laboratório (WHO, 1997). Nestes estudos, a dose de um composto na qual nenhum efeito adverso foi observado nos animais é determinada (NOAEL) e a este valor se aplica um fator de segurança para se derivar a IDA. Este fator de segurança, normalmente 100 , corresponde à variação de sensibilidade entre a espécie testada e o homem (10 vezes mais sensível) e à variação de sensibilidade dentro da população humana (grupos 10 vezes mais sensíveis que a média da população). IDA de vários pesticidas são estabelecidas e publicadas pela FAO/OMS e agências governamentais como o EPA (US Environmental Protection Agency). Valores estabelecidos até hoje para diversos compostos variam entre 0,0003 e $5 \mathrm{mg} / \mathrm{Kg}$ de peso corpóreo (CODEX, 2000). Em 2001, o Brasil passou a incluir os valores de IDA nas monografias de pesticidas aprovados no país (ANVISA, 2002).

\section{Avaliação do Risco Crônico}

Para se avaliar o risco crônico a que o homem está exposto, quando ingere alimento contaminado com agroquímico, é necessária a condução de um estudo de avaliação de risco. Neste estudo, a ingestão diária total de cada pesticida, em $\mathrm{mg} / \mathrm{dia}$, é calculada pela soma das ingestões do pesticida pelo consumo de cada alimento da dieta. Para cada alimento, a ingestão é calculada multiplicado o consumo do alimento (A) pela concentração do pesticida no alimento (P). Desta maneira, para cada pesticida,

\section{Ingestão total $=S(A \times P)$}

Para se avaliar o risco que esta ingestão pode representar para a saúde humana, compara-se a ingestão total calculada com a ingestão diária aceitável (IDA). Risco pode existir quando a ingestão total ultrapassa a IDA (\% IDA>100) (WHO, 1997). 
$\%$ IDA = Ingestão total $\times 100 / I D A$

Valores de concentração do pesticida utilizados no cálculo da ingestão total devem refletir o nível de concentração do composto, no momento do consumo. Todavia, quando estes valores não estão disponíveis, valores de limite máximo de resíduos (LMR), estabelecidos pelos órgãos internacionais e nacionais para cada binômio pesticida/cultura, podem ser utilizados como parâmetro de concentração. Esta metodologia, porém, é altamente conservadora já que assume o consumo diário de todos os alimentos para os quais existe uso aprovado e que os níveis de resíduos no alimento consumido são iguais aos LMR (WHO, 1997).

Até 1997, o Grupo de Peritos em Resíduos de Pesticidas da FAO/OMS (JMPR) conduzia o estudo de avaliação de risco, utilizando os LMR como parâmetros de concentrações. Em 1996, o Grupo introduziu o conceito de nível mediano de resíduo (STMR), que reflete melhor o nível de resíduos de pesticidas no alimento tratado, e passou a usar este valor no estudo de avaliação de risco a partir de 1998. Dos 26 compostos avaliados pelo JMPR em 2000 e 2001, apenas dois compostos (fenitrotion e haloxifop) tiveram sua ingestão ultrapassando a IDA.

Num estudo de avaliação de risco, conduzido recentemente em 281 agroquímicos com uso aprovado no país, utilizando os LMR como concentrações de resíduos, 23 compostos apresentaram porcentagem da IDA>100 em pelo menos uma dieta regional brasileira (Caldas \& Souza, 2000). Destes pesticidas, 16 eram inseticidas organofosforados e 3 fungicidas ditiocarbamatos. De maneira a se conduzir o refinamento deste estudo e avaliar o risco real da exposição crônica da população brasileira aos agroquímicos, é necessário gerar dados de concentração de resíduos que melhor reflitam a ingestão destes compostos no alimento, no momento do consumo.

\section{Avaliação da Exposição Humana}

Um estudo da avaliação da exposição humana aos fungicidas ditiocarbamatos por meio da ingestão de tomate foi conduzido para um total de 180 amostras coletadas pelo Departamento de Fiscalização e Saúde do Distrito Federal, no período de seca e chuva de 1998 e 1999 e seca e chuva de 1999 / 2000 (Conceição, 2002). A avaliação da exposição humana a esses compostos foi calculada, utilizando os dados de resíduos de ditiocarbamatos em tomates obtidos nos períodos de seca e chuva e de consumo alimentar de tomate no Distrito Federal $(0,018 \mathrm{~kg} /$ dia) (IBGE, 1999), segundo a equação abaixo (WHO, 1997).

Ingestão $(\mathrm{mg} /$ pessoa $/$ dia $)=[$ ditiocarbamato $(\mathrm{mg} / \mathrm{kg})]$ X 0,018 $\mathrm{kg} /$ dia

Os resultados do cálculo da ingestão estão mostrados na Tabela 1. 
Tabela 1. Ingestão de fungicidas ditiocarbamatos pelo consumo de tomate.

\begin{tabular}{ccc}
\hline Período & $\begin{array}{c}\text { Resíduos de } \\
\text { ditiocarbamatos }(\mathrm{mg} \\
\text { CS2 } / \mathrm{kg}) \mathrm{a}\end{array}$ & $\begin{array}{c}\text { Ingestão diária(mg } \\
\text { /pessoa/dia) }\end{array}$ \\
\hline Seca de 1998 & 0,15 & 0,0027 \\
Chuva de 1999 & 0,34 & 0,0061 \\
Seca de 1999 & 0,23 & 0,0041 \\
Chuva de 2000 & 0,25 & 0,0045 \\
\hline
\end{tabular}

$a=$ média aritmética dos resíduos no período do estudo.

Para se avaliar o risco desta exposição para a saúde humana, compara-se esta ingestão com o parâmetro toxicológico, a ingestão diária aceitável (IDA). Riscos podem existir quando a ingestão ultrapassa a IDA. Os valores de IDA, que são individuais para cada ditiocarbamato, podem ser calculados em termos de $\mathrm{CS}_{2}$, composto liberado na hidrólise do ditiocarbamato, utilizando o fator de correção molecular para cada composto, e expressos em mg/pessoa/dia (Tabela 2).

Tabela 2. IDA para os fungicidas ditiocarbamatos.

\begin{tabular}{ccccc}
\hline Pesticida & $\begin{array}{c}\text { IDAa(mg/k- } \\
\text { g pc/dia) }\end{array}$ & $\begin{array}{c}\text { Fator de } \\
\text { correção }\end{array}$ & $\begin{array}{c}\text { IDA } \\
\text { (mg/kg } \\
\text { pc/dia) CS2 }\end{array}$ & $\begin{array}{c}\text { IDAb(mg/- } \\
\text { pessoa/dia }) \\
\text { CS2 }\end{array}$ \\
\hline Ziram & 0,003 & 2,01 & 0,0015 & 0,09 \\
Mancozeb & 0,006 & 1,78 & 0,0034 & 0,20 \\
Maneb & 0,005 & 1,74 & 0,0029 & 0,17
\end{tabular}

a. Codex Alimentarius e EPA; b. considerando o peso médio de uma pessoa igual a $60 \mathrm{~kg}$ Universitas Ciências da Saúde - vol.01 n.01 - pp.97-103 
Os níveis de resíduos obtidos neste estudo refletem o uso de qualquer ditiocarbamato. Para a avaliação de risco, considera-se a menor IDA, em $\mathrm{CS}_{2}$, entre os compostos, isto é $0,09 \mathrm{mg} /$ pessoa/dia para o ziram. Para cada período do estudo, podemos calcular a contribuição da ingestão de tomate para a dose diária aceitável, \% IDA (Tabela 3).

$\%$ IDA $=($ Ingestão $/ 0,09 \mathrm{mg} /$ pessoa /dia $) \times 100$

Tabela 3. Avaliação de risco do consumo de ditiocarbamatos pela ingestão de tomate

\begin{tabular}{ccc}
\hline Período & $\begin{array}{c}\text { Ingestão(mg } \\
\text { /pessoa/dia) }\end{array}$ & \% IDA \\
\hline Seca de 1998 & 0,0027 & 3,0 \\
Chuva de 1999 & 0,0061 & 6,4 \\
Seca de 1999 & 0,0041 & 4,6 \\
Chuva de 2000 & 0,0045 & 5,0 \\
\hline
\end{tabular}

\section{Conclusão}

Os resultados da Tabela 3 mostram que a ingestão de resíduos de ditiocarbamatos, mediante o consumo de tomate pela população do DF variou de 3,0 a 6,4\% da IDA. Os ditiocarbamatos são permitidos pela legislação brasileira para outras culturas além do tomate, incluindo as frutas, cereais e outras hortaliças; mancozeb é permitido para uso em 33 culturas; maneb em 26 culturas e ziram em 9 culturas (ANVISA, 2001). Desta maneira, é importante ressaltar que a ingestão de ditiocarbamatos na dieta brasileira pode ser significativamente maior que a contribuição dada apenas pelo consumo de tomate. 


\section{Referências bibliográficas}

ANVISA. AGÊNCIA NACIONAL DE VIGILÂNCIA SANITÁRIA. TOXICOLOGIA. Disponível em URL: http://www.anvisa.gov.br, 2002.

BONSALL, J.L. Measurement of occupational exposure to pesticide. In: Occupational Hazards of Pesticides Use. Ed. Turnbull, G.S.; Francis and Taylor, London, pp. 13-33, 1985.

CALDAS, E.D; SOUZA, L.C.R.K. Avaliação do de risco crônico da ingestão de resíduos de pesticidas na dieta brasileira, Revista de Saúde Pública, 34, 529-37, 2000.

CODEX ALIMENTARIUS. Pesticide Residues In Food-Maximum residue limits. v. 2 B. Food and Agriculture Organization of the United Nations, World Health Organization. Rome, 2000.

CONCEIÇÃO, M.H. Resíduos de Pesticidas em Tomates: Metodologia Analítica e Avaliação da Exposição Humana, Tese de Doutorado, UnB, Brasília, 2002.

DOLL R.; PETTO, R. The causes of cancer: quantitative estimates of avoidable risks of cancer in the United States Today., J. Natl. Cancer Inst., 66, 1191-1308, 1981.

ECOBICHON, D.J. In: Casarett \& Doull's; Toxicology - The basic science of poisons. Ed. Mary Amdur, John Doull, Curtis, D. Klaassen. $5^{\text {th }}$ Ed., Mc Graw-Hill, 1996.

IBGE. Instituto Brasileiro de Geografia e Estatística. Pesquisa de Orçamentos Familiares 1995-1996. Consumo alimentar domiciliar per capita anual,. 1999.

JEYARATNAM, J. Occupational health issues in development countries. In: Organização Mundial da Saúde. Public Health impact of pesticides used in agriculture, Geneva, 207-212, 1990.

SINITOX, Sistema Integrado de informação Toxicológica. Fundação Oswaldo Cruz; disponível-URL:http://www.fiocruz.br.cict.oque.estrut.dect/sintox.kitbrasil.html, pesquisa realizada em janeiro de 2002.

WHO, Guidelines for predicting dietary intake of pesticide residues, World Health Organization - WHO, Geneva, 1997.

WHO. Disponível. URL: http://www.cdc.gov/niosh/pestsurv/default.html\#mmwr, janeiro de 2002.

WINTER, C. Dietary pesticide risk assessment, Rev. Eniron. Cont. Tox., 127, 23-67, 1992.

ZAMBRONE, F.A.D. URL: http://www.hc.unicamp.br/, pesquisa feita em 1999. 